\title{
Gibbs energy of superoxide dismutase heterodimerization accounts for variable survival in amyotrophic lateral sclerosis
}

\author{
Yunhua Shi $^{\mathrm{a} \ddagger}$, Mark J. Acerson ${ }^{\mathrm{a}}$, Alireza Abdolvahabi ${ }^{\mathrm{a}}$, Richard A. Mowery ${ }^{\mathrm{a}}$, Bryan F. Shaw ${ }^{\mathrm{a}}$ \\ ${ }^{\mathrm{a}}$ Department of Chemistry and Biochemistry, Baylor University, Waco, TX 76798-7348
}

To whom correspondence should be addressed: Bryan_Shaw@baylor.edu

${ }^{\ddagger}$ These authors contributed equally to this work.

***Supporting Information*** 


\section{Derivation of rate law for different heterodimerization mechanisms:}

We have performed a derivation of the rate laws for all three proposed mechanisms (see Figure 1 of the main text) using steady-state assumptions for each intermediate. Mechanisms 1 and 2 predict half-order kinetics for WT and mutant (ALS), i.e., first-order overall. In contrast, the rate law for mechanism 3 yields first-order kinetics with respect to WT and half-order for ALS (1.5order overall).

This kinetic rationale is dependent upon steady-state approximation for the intermediate, whether it is a monomer or tetramer (or some other oligomeric intermediate). Steady-state approximations are likely to apply in this instance because the proposed intermediates maintain a low and relatively constant concentration during equilibration (i.e., intermediates are not observed during CE measurements and are known to exist in low concentrations under physiological conditions ${ }^{1,2}$ ).

\section{Rate Law Derivation for Mechanism 1:}

Mechanism 1 proposes that heterodimer formation occurs through the association of one WT and one ALS monomer, i.e., through a pathway that is analogous to $\mathrm{S}_{\mathrm{N}} 1$ mechanisms in organic chemistry and dissociative reactions in inorganic chemistry.

$$
W T_{\text {mon }}+A L S_{\text {mon }} \underset{k_{-1}}{\stackrel{k_{1}}{\rightleftharpoons} W T A L S_{H e t}}
$$

The rate expression for the above equilibrium is:

$$
\frac{d\left[W T A L S_{H e t}\right]}{d t}=k_{1}\left[W T_{\text {mon }}\right]\left[A L S_{\text {mon }}\right]-k_{-1}\left[W T A L S_{H e t}\right]
$$

Given that we are unable to measure the concentrations of $\mathrm{WT}_{\text {mon }}$ and $\mathrm{ALS}_{\text {mon }}$ using $\mathrm{CE}$, we express the rate of heterodimerization in terms of $\left[\mathrm{WT}_{\mathrm{Hom}}\right]$ and $\left[\mathrm{ALS}_{\mathrm{Hom}}\right]$. 
WT SOD1 and its related mutants are known to favor dimeric versus monomeric states, as indicated by reported dimer dissociation $\left(\mathrm{K}_{\mathrm{d}}\right)$ values of $\sim 10 \mathrm{nM}$ for WT, and between 10-1100 $\mathrm{nM}$ for various ALS mutants. ${ }^{2,3}$ Therefore, the physiologically relevant concentration of SOD1 used in our experiments $(\sim 50 \mu \mathrm{M})$ allows for a minor fraction of WT or ALS SOD1 to populate its monomeric state $\left(\sim 2-10 \%^{2}\right)$ with the majority of SOD1 remaining dimeric.

$$
\begin{array}{r}
W T_{\text {mon }} \underset{k_{-2}}{\stackrel{k_{2}}{\rightleftharpoons}} \frac{1}{2} W T_{\text {Hom }} \\
M U_{\text {mon }} \underset{k_{-3}}{\stackrel{k_{3}}{\rightleftharpoons}} \frac{1}{2} A L S_{\text {Hom }}
\end{array}
$$

The above reactions quickly reach to steady-state because $\left[\mathrm{WT}_{\text {mon }}\right]$ and $\left[\mathrm{ALS}_{\text {mon }}\right]$ are small at equilibrium. $\left[\mathrm{WT}_{\mathrm{mon}}\right]$ and $\left[\mathrm{ALS}_{\mathrm{mon}}\right]$ can then be related to their dimeric concentrations as follows:

$$
\begin{aligned}
k_{2}\left[W T_{\text {mon }}\right] & =k_{-2}\left[W T_{\text {Hom }}\right]^{1 / 2} \\
k_{3}\left[A L S_{\text {mon }}\right] & =k_{-3}\left[A L S_{\text {Hom }}\right]^{1 / 2}
\end{aligned}
$$

These expressions can be solved for $\left[\mathrm{WT}_{\mathrm{mon}}\right]$ and $\left[\mathrm{ALS}_{\mathrm{mon}}\right]$ respectively:

$$
\begin{gathered}
{\left[W T_{\text {mon }}\right]=\frac{k_{-2}}{k_{2}}\left[W T_{\text {Hom }}\right]^{1 / 2}} \\
{\left[A L S_{\text {mon }}\right]=\frac{k_{-3}}{k_{3}}\left[A L S_{\text {Hom }}\right]^{1 / 2}}
\end{gathered}
$$

Steady-state approximation (i.e., an intermediate is formed in low concentration and the intermediate concentration remains essentially constant throughout the course of the reaction) can thus be used to justify the substitution of our solved expressions for $\left[\mathrm{WT}_{\mathrm{mon}}\right]$ and $\left[\mathrm{ALS}_{\mathrm{mon}}\right]$ in terms of $\left[\mathrm{WT}_{\mathrm{Hom}}\right]$ and $\left[\mathrm{ALS}_{\mathrm{Hom}}\right]$ into our initial rate expression: 


$$
\frac{d\left[W T A L S_{H e t}\right]}{d t}=k_{1} \frac{k_{-2}}{k_{2}}\left[W T_{H o m}\right]^{1 / 2} \frac{k_{-3}}{k_{3}}\left[A L S_{H o m}\right]^{1 / 2}-k_{-1}\left[W T A L S_{H e t}\right]
$$

Grouping rate constants into a single $k, k_{4}$, simplifies the above rate equation to:

$$
\frac{d\left[W T A L S_{H e t}\right]}{d t}=k_{4}\left[W T_{H o m}\right]^{1 / 2}\left[A L S_{H o m}\right]^{1 / 2}-k_{-1}\left[W T A L S_{H e t}\right]
$$

Where

$$
k_{4}=\frac{k_{1} k_{-2} k_{-3}}{k_{2} k_{3}}
$$

This rate expression can be simplified further when considering that the initial concentration of WTALS $_{\text {Het }}$ is equal to zero at $\mathrm{t}=0$. This assumption reduces the final term in the rate expression to zero, leaving the final rate law for mechanism 1 at $t=0$ to be:

$$
\frac{d\left[W T A L S_{H e t}\right]_{i}}{d t}=k_{4}\left[W T_{H o m}\right]_{i}^{1 / 2}\left[A L S_{H o m}\right]_{i}^{1 / 2}
$$

According to the solved rate expression for mechanism 1, the formation of WTALS $\mathrm{Het}_{\mathrm{H}}$ should only depend on the concentration of $\mathrm{WT}_{\mathrm{Hom}}$ and $\mathrm{ALS}_{\mathrm{Hom}}$ with half-order kinetics for each SOD1 protein.

\section{Rate Law Derivation for Mechanism 2:}

Mechanism 2 proposes heterodimer formation to occur through a heterotetramer intermediate being composed of two WT and two ALS monomers, i.e., through a pathway that is analogous to $\mathrm{S}_{\mathrm{N}} 2$ mechanisms in organic reaction mechanisms, and associative reactions in inorganic reaction mechanisms. The basis for assuming a tetrameric SOD1 intermediate is supported by previous observations of tetrameric SOD1 using atomic force microscopy (AFM) on homogenized solutions of WT and A4V mutant SOD1. ${ }^{1}$ 


$$
\frac{1}{2} W_{T A L S} \stackrel{k_{\text {tet }}}{\stackrel{k_{5}}{\rightleftharpoons}} W_{k_{-5}} \text { TALS }_{\mathrm{Het}}
$$

The rate expression for the above equilibrium is:

$$
\frac{d\left[W T A L S_{H e t}\right]}{d t}=k_{5}\left[W T A L S_{t e t}\right]^{1 / 2}-k_{-5}\left[W T A L S_{H e t}\right]
$$

Similar assumptions can be made for the distribution of SOD1 between dimeric and tetrameric states as in mechanism 1 for dimeric and monomeric states. Rapid equilibration occurs once again between the dimeric and tetramic states of WT and ALS SOD1 given that [WTALS tet $_{\text {is }}$ small at equilibrium.

$$
W T_{\text {Hom }}+A L S_{\text {Hom }} \underset{k_{-6}}{\stackrel{k_{6}}{\rightleftharpoons} W T A L S_{\text {tet }}}
$$

Relating $\left[\mathrm{WT}_{\mathrm{Hom}}\right]$ and $\left[\mathrm{ALS}_{\mathrm{Hom}}\right]$ to $\left[\mathrm{WTALS}_{\mathrm{tet}}\right]$ at equilibrium using the above expression yields:

$$
k_{-6}\left[W T A L S_{\text {tet }}\right]=k_{6}\left[W T_{\text {Hom }}\right]\left[A L S_{\text {Hom }}\right]
$$

This gives the expression for $\left[\mathrm{WTALS}_{\mathrm{tet}}\right]$ as:

$$
\left[W T A L S_{\text {tet }}\right]=\frac{k_{6}}{k_{-6}}\left[W T_{\text {Hom }}\right]\left[A L S_{\text {Hom }}\right]
$$

Applying steady-state approximation and substituting the solved equation for $\left[\mathrm{WTALS}_{\text {tet }}\right.$ into the rate expression allows the conversion to observable species:

$$
\frac{d\left[W T A L S_{H e t}\right]}{d t}=k_{5}\left(\frac{k_{6}}{k_{-6}}\left[W T_{\text {Hom }}\right]\left[A L S_{H o m}\right]\right)^{1 / 2}-k_{-5}\left[W T A L S_{H e t}\right]
$$

Grouping rate constants into a single $k, k_{7}$, simplifies the above rate equation to:

$$
\frac{d\left[W T A L S_{H e t}\right]}{d t}=k_{7}\left[W T_{H o m}\right]^{1 / 2}\left[A L S_{H o m}\right]^{1 / 2}-k_{-5}\left[W T A L S_{H e t}\right]
$$

Where 


$$
k_{7}=\frac{k_{5} k_{6}^{1 / 2}}{k_{-6}^{1 / 2}}
$$

Applying this simplified rate equation only to initial rates at $\mathrm{t}=0$ implies that $\left[\mathrm{WTALS}_{\text {tet }}\right]$ at $\mathrm{t}=$ 0 equals zero.

The rate expression at $\mathrm{t}=0$ thus reduces to:

$$
\frac{d\left[W T A L S_{\text {Het }}\right]_{i}}{d t}=k_{7}\left[W T_{\text {Hom }}\right]_{i}^{1 / 2}\left[A L S_{\text {Hom }}\right]_{i}^{1 / 2}
$$

This predicted rate expression for the formation of WTALS $\mathrm{Wet}_{\mathrm{H}}$ is indistinguishable from that derived for $\mathrm{WTALS}_{\mathrm{Het}}$ formation through monomerization, i.e., mechanism 1, which indicates that our current method of measuring heterodimerization with $\mathrm{CE}$ is unable to distinguish between mechanisms 1 and 2 because the reaction proceeds via half-order dependence on both [WT $\left.\mathrm{Wom}_{\mathrm{Hom}}\right]$ and $\left[\mathrm{ALS}_{\mathrm{Hom}}\right]$. Moreover, CE lacks the ability to measure either of the proposed intermediates, either monomer or heterotetramer concentrations, as a function of time. The reasons for our inability to measure these species have already been described in the main text.

\section{Rate Law Derivation for Mechanism 3:}

Mechanism 3 proposes that heterodimerization occurs through an intermediate consisting of a 2:1 ratio of WT to ALS or vice versa. Here, we will only derive one of these scenarios (monomeric ALS with dimeric WT) because both produce the same ratio of dependence on WT and ALS (either 1:0.5 or $0.5: 1$ ).

$$
W T_{2} A L S_{\text {tri }} \stackrel{k_{8}}{\rightleftharpoons} W T A L S_{\text {Het }}+W T_{\text {mon }}
$$

The rate expression for the above equilibrium is:

$$
\frac{d\left[W T A L S_{H e t}\right]}{d t}=k_{8}\left[W T_{2} A L S_{t r i}\right]-k_{-8}\left[W T A L S_{H e t}\right]\left[W T_{m o n}\right]
$$


The trimeric intermediate is not observed by $\mathrm{CE}$ and should have a unique mobility and different from all other species previously discussed. This implies two possibilities: (i) that trimers do not form and degrade faster than the timescale of $\mathrm{CE}$, or (ii) concentration of the trimeric intermediate remains low throughout the course of the reaction as previously assumed for both monomeric and tetrameric intermediates. Based on these assumptions and similar to those applied in the derivation of rate laws for mechanisms 1 and 2, equilibrium between dimeric WT and monomeric ALS SOD1 is achieved as:

$$
W T_{\text {Hom }}+A L S_{\text {mon }} \stackrel{k_{9}}{\rightleftharpoons} W T_{2} A L S_{\text {tri }}
$$

$\left[\mathrm{WT}_{2} \mathrm{ALS}_{\text {tri }}\right]$ can then be related to $\left[\mathrm{WT}_{\mathrm{Hom}}\right]$ and $\left[\mathrm{ALS}_{\mathrm{mon}}\right]$ :

$$
k_{-9}\left[W T_{2} A L S_{\text {tri }}\right]=k_{9}\left[W T_{\text {Hom }}\right]\left[A L S_{\text {mon }}\right]
$$

Solving for $\left[\mathrm{WT}_{2} \mathrm{ALS}_{\text {tri }}\right]$ :

$$
\left[W T_{2} A L S_{\text {tri }}\right]=\frac{k_{9}}{k_{-9}}\left[W T_{H o m}\right]\left[A L S_{\text {mon }}\right]
$$

And substituting into the rate law above gives:

$$
\frac{d\left[W T A L S_{H e t}\right]}{d t}=k_{8} \frac{k_{9}}{k_{-9}}\left[W T_{H o m}\right]\left[A L S_{\text {mon }}\right]-k_{-8}\left[W T A L S_{H e t}\right]\left[W T_{\text {mon }}\right]
$$

$\left[\mathrm{ALS}_{\mathrm{mon}}\right]$ was previously solved in terms of $\left[\mathrm{ALS}_{\mathrm{Hom}}\right]$ and can be substituted as:

$$
\frac{d\left[W T A L S_{H e t}\right]}{d t}=k_{8} \frac{k_{9}}{k_{-9}}\left[W T_{H o m}\right] \frac{k_{-3}}{k_{3}}\left[A L S_{H o m}\right]^{1 / 2}-k_{-8}\left[W T A L S_{H e t}\right]\left[W T_{m o n}\right]
$$

Grouping rate constants into a single $k, k_{10}$, simplifies the above rate equation to:

$$
\frac{d\left[W T A L S_{H e t}\right]}{d t}=k_{10}\left[W T_{H o m}\right]\left[A L S_{H o m}\right]^{1 / 2}-k_{-8}\left[W T A L S_{H e t}\right]\left[W T_{\text {mon }}\right]
$$

Where 


$$
k_{10}=\frac{k_{8} k_{9} k_{-3}}{k_{-9} k_{3}}
$$

At $\mathrm{t}=0,\left[\mathrm{WTALS} \mathrm{Set}_{\mathrm{Het}}\right]=0$. The rate equation at $\mathrm{t}=0$ finally simplifies to

$$
\frac{d\left[W T A L S_{H e t}\right]_{i}}{d t}=k_{10}\left[W T_{H o m}\right]_{i}\left[A L S_{H o m}\right]_{i}^{1 / 2}
$$

This theoretical rate expression does not agree with our observed overall first-order kinetics for the formation of heterodimer, which makes mechanism 3 invalid.

\section{Supporting Figures:}

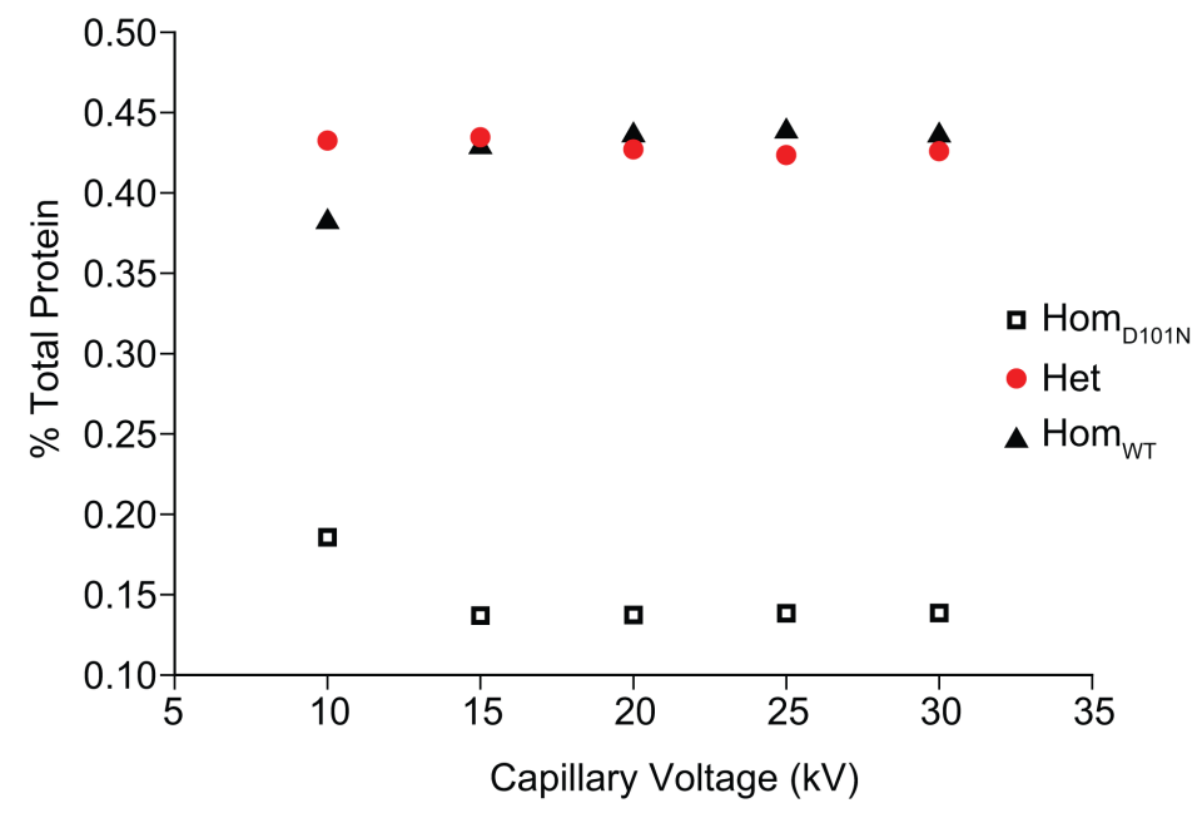

Figure S1. Variations in capillary voltage do not significantly alter the kinetics of heterodimerization between WT apo-SOD1 and D101N apo-SOD1.

\section{Supporting References:}

(1) Allen, M. J.; Lacroix, J. J.; Ramachandran, S.; Capone, R.; Whitlock, J. L.; Ghadge, G. D; Arnsdorf, M. F.; Roos, R. P.; Lal, R. Neurobiol. Dis. 2012, 45, 831-838.

(2) McAlary, L.; Yerbury, J. J.; Aquilina, J. A. Sci. Rep. 2013, 3, 3275.

(3) Svensson, A. E.; Osman, B.; Kayatekin, C.; Adefusika, J. A.; Zitzewitz, J. A.; Matthews, C. R. PLoS ONE 2010, 5, e10064.

(4) Zubriene, A.; Matuliene, J.; Baranauskiene, L.; Jachno, J.; Torresan, J.; Michailoviene, V; Cimmperman, P.; Matulis, D. Int J. Mol. Sci. 2009, 10, 2662-2680. 